\title{
Heterogeneous HPC Environments
}

\author{
Marco Vanneschi \\ Department of Computer Science, University of Pisa, Italy
}

\begin{abstract}
Directions of software technologies for innovative HPC environments are discussed according to the industrial user requirements for heterogeneous multidisciplinary applications, performance portability, rapid prototyping and software reuse, integration and interoperability of standard tools. The various issues are demonstrated with reference to the PQE2000 project and its programming environment SkIE (Skeleton-based Integrated Environment). Modules developed by a variety of standard languages and tools are encapsulated into SkIECL (SkIE Coordination Language) structures to form the global application. A performance model associated to SkIECL allows the static and dynamic tools to introduce a large amount of global optimizations without the direct intervention of the programmer. The paper discusses also some of the most critical issues in matching architecture and software technology, showing how the SkIE environment, and its evolutions, can act as a solid framework in which innovative hardware-software systems for HPC can be studied and experimented.
\end{abstract}

\section{Introduction}

Broadening the use of High Performance Computing beyond its current research applications requires correction of some shortcomings in the technology at all levels: hardware architecture, software environments, and their relationships. A very critical role is played by the software technology of integrated programming environments for the modular and efficient development of industrial and commercial applications. Traditionally, the target of research and development in HPC has been mainly directed towards the scientific users and the Grand Challenge applications. However, it is important to recognize [1] that the widespread diffusion of HPC technology depends on its ability to satisfy the needs of industrial users: their main goal is to exploit HPC technology to significantly reduce the time-to-market of products. In the same way, HPC must be able to satisfy the requirements for critical public services and government decision making strategies.

These requirements represent the basic goals of the PQE2000 project [3]. PQE2000 is a joint initiative of the main Italian research agencies - CNR, ENEA, INFN - and of Finmeccanica's QSW (Quadrics Supercomputers World Ltd) for the realization of innovative HPC general-purpose systems and their applications for industry, commerce and public services. As a consequence of PQE2000 emerging technology, a Research Programme on HPC has been proposed aiming 
at the definition of a large European initiative in HPC technology and applications. The results of PQE2000 activities are transferred into industrial products by QSW, as in the case of the programming environment discussed in this paper. A research road map towards PetaFlops architectures and programming environments has been established and, at the same time, a first innovative version of PQE2000 technology has been realized by integrating stable existing products. In this paper, the basic ideas on the technologies for innovative HPC software environments are discussed and described with reference to the PQE2000 project and its programming environment SkIE (Skeleton-based Integrated Environment) [2,4]. In SkIE an application is developed with the assistance of a coordination language (SkIECL), based on the skeletons model [9-11,5,12], in order to integrate, and to globally optimize the usage, of independent software modules: these last are, possibly very complex, programs independently developed by existing standard languages (host languages) and tools. Currently, the host languages include $\mathrm{C}, \mathrm{C}++, \mathrm{F} 77, \mathrm{~F} 90$, and HPF. Work is in progress to integrate Java and other standard object-oriented tools. The coordination language SkIECL is an industrial version of $P^{3} L$, a prototype research vehicle designed at the Department of Computer Science, University of Pisa [5-8]. SkJECL allows the software designers to express, in a primitive and structured way, both data parallelism and task parallelism. Such structures can be combined hierarchically at any depth according to the principles for modular software development. The variety of parallelism strategies and their structured composition are the key issues for achieving global optimizations in parallel programs and to overcome the typical difficulties and inefficiencies of SPMD/data parallel languages. In section 2 we discuss the basic issues in the development of heterogeneous HPC environments. Section 3 contains a description of the SkIE programming model. Section 4 is dedicated to the discussion of critical issues in matching architecture and software technology.

\section{Heterogeneous Multidisciplinary Environments}

The introduction of software engineering methodologies in HPC global applications development requires that the current "in the small" approach evolves rapidly towards "in the large" development tools and environments. A parallel application is not merely a parallel algorithm: the parallelization problems must be solved along with the efficient integration of heterogeneous objects such as existing software modules, files and data base management tools, visualization tools, application libraries and packages. More in general, a comprehensive solution to the industrial needs in HPC application development implies to deal with several heterogeneity issues as discussed in the following.

Multidisciplinary Environments. Multidisciplinary environments for parallel machines are needed, by which an entire development team can easily take part in a complex industrial project. As an example, in many industrial areas e.g. motor-car, energy and environment, aerospace, biotechnology and medicine, 
finance, new media services - application development implies, besides the utilization of different specific simulation techniques, the exploitation of techniques and tools for data mining, virtual reality, multipurpose analysis and optimization, interactive visualization, and so on. Achieving the goal of multidisciplinary applications requires the realization of integrated programming environments for parallel machines. As in the general context of Information Technology, this means that all the current and future standard languages, tools and packages for application design, each of which is possibly specialized towards an application area, are made available in the context of a uniform and well-structured development environment. However, the integration must also be characterized by high performance and efficiency, and this is much more important for multidisciplinary applications aiming to improve the industrial time-to-market and productivity: often, the combined utilization of several different tools, to form a multidisciplinary application, needs high performance systems, since it involves the execution of large amounts of tasks, each one structurable as a (massively) parallel program. The goal of high performance in multidisciplinary applications cannot be achieved by low-level machine-dependent mechanisms and tools and by mere "porting/rewriting" techniques: high-level, automatic tools must be available in order to restructure and to optimize a, possibly very large, set of heterogeneous modules individually developed by means of different standard languages and tools.

Performance Portability. Because of the requirements for reliability and standardization, as well as the continuous evolution of systems and of simulation techniques, industry privileges the utilization of commercial, general purpose systems and products that guarantee the portability of applications across different machine platforms. This is especially needed in a situation in which a great deal of uncertainty exists among industrial users about the characteristics of the next generation machines. Thus, the development environment we advocate is a high-level, multidisciplinary, multi-platform one. All the known HPC platforms are of interest: distributed memory and shared memory MPP, SMP, clusters of SMP, PIM, combined architectures (e.g. MPP+SMP, MPP+PIM), as well as metacomputers. Moreover, the programming environment must be able to address the effective utilization of future PetaFlops machines.

Especially in HPC, portability implies performance portability, i.e. the ability of a programming environment to support restructuring and re-optimization of applications passing from one machine platform to another one, or simply from one configuration/model of the same machine to the next one. This has to be done mainly in a (semi-)automatic manner, by means of powerful restructuring compiler tools and/or by suitable run-time supports. In SkIE the run-time support consists of a collection of very efficient implementation templates (fully invisible to the programmer), where more than one template exists for each skeleton with respect to the variety of underlying platforms. The design of implementation templates is done on top of a standard, portable abstract machine: an MPI machine has been adopted in the current version. 
Rapid Prototyping and Software Reuse. The programming environment must allow the design team to develop new applications by a rapid prototyping approach. In successive design phases the application prototype could be refined and restructured for the sake of performance and/or modularity and reliability, by means of explicit and/or semiautomatic techniques. Very often, this feature implies the ability to reuse existing software modules or objects, without changing them, i.e. the ability to encapsulate existing modules into structured parallel programs, or with minimum modifications. This concept should be applied to modules written in any sequential language or even to already parallelized modules. Analyzing the software reuse feature in terms of achievable performance, two approaches to parallel program optimization can be distinguished :

- global optimizations, in which a computation is viewed as a parallel collection of modules, each of which is considered as a "black box" : optimizations are done at the level of the whole structure, without affecting the optimizations done by the native compiler of the imported modules;

- local optimizations, in which new opportunities for parallelism detection are exploited inside the modules according to the characteristics of the whole application and/or of the underlying machine.

Global optimizations are fundamental for rapid prototyping and software reuse, with the performance portability goal in mind. However, in terms of performance refinement, software reuse may imply also the adoption of local optimizations of the imported modules. Currently, in SkIE only global optimizations are implemented, relying on the existing standard compilers for the local optimizations. Research is in progress along the line of global + local optimizations: a notable example is the optimization of HPF modules in the context of a skeleton global structure that includes task parallelism [13].

\section{Programming Models for Heterogeneous Environments}

\subsection{Modular HPC Environments}

The issues discussed in sect. 2 emphasize the viewpoint that, despite several current approaches, HPC methodologies and tools should fully respect the Computer Science principles of modular software development. We believe that, even in this context, it is feasible to achieve high performance and performance portability all the same, i.e. the find satisfactory solutions to the so called PPP (Programmability, Portability, Performance) problem.

Principles of modular software development have been often, e.g. [17], enunciated by J. Dennis: information hiding: the internal structure of a module must not be known to the user; invariant behavior: the functional behavior of a module must be independent of the context in which it is invoked; data generality: it must be possible to pass any data object onto the interface to a module; secure arguments: side-effects on arguments must not be allowed at the interface of a module; recursive construction: any, possibly complex, computation must be usable as a module in building larger computations; system resource management: 
resource, e.g. storage and communication, management must be performed by the system, invisibly to the program modules.

\subsection{The Coordination Language Approach}

The requirements stated till now have led to the idea of a coordination language to design complex HPC applications. The constructs of the coordination language must allow the designer to express the global structure of the parallel application in a high-level, fully machine independent fashion (in-the-large development of HPC programs). The constructs represent the only forms of parallelism (mechanisms + strategies) that can be used to express a parallel computation. Independently developed modules are easily inserted in to the global application structure according to the rules for combining and interfacing the parallel constructs.

The programming model of SkIE, i.e. the coordination language SkIECL of the integrated development environment, is based on the skeletons model [9-11, $5,12]$. Potentially, the skeletons approach has all the interesting properties advocated in the previous sections. In fact, some of the proposed skeletons systems are characterized by the compositionality property and by the existence of a formal performance model to drive the compiler optimizations: the term structured parallel programming has been introduced to denote those skeletons models that possess such powerful features. Among them : BMF [20], SCL [10], BACS [21] and $P^{3} L[5]$. In SkIECL the following skeletons (second order functions) have been chosen for the parallel constructs:

- stream parallel skeletons:

seq: sequential module with well defined in-out interfaces

pipe: pipelined composition of skeletons

farm: self-load balancing process farm, where the generic (functionally replicated) worker can be any skeleton

loop: data-flow loop, where the body can be any skeleton

- data parallel skeletons:

map: independent data-parallel computations without communication reduce: parallel reduction by binary associative and commutative operators comp: sequential function composition to express data parallel computation with communication (stencils)

Figure 1 and 2 illustrate the graphical representation and the textual syntax of stream parallel skeleton and data parallel skeleton respectively. Specific issues to understand the approach are discussed in the following.

Compositionality. Compositionality of the coordination language constructs is, of course, fundamental to build modular program structures and, at the same time, to exploit the desired degree of parallelism. Figure 3 shows a SkJECL program computing a ray tracing algorithm on stream of images. In particular the 


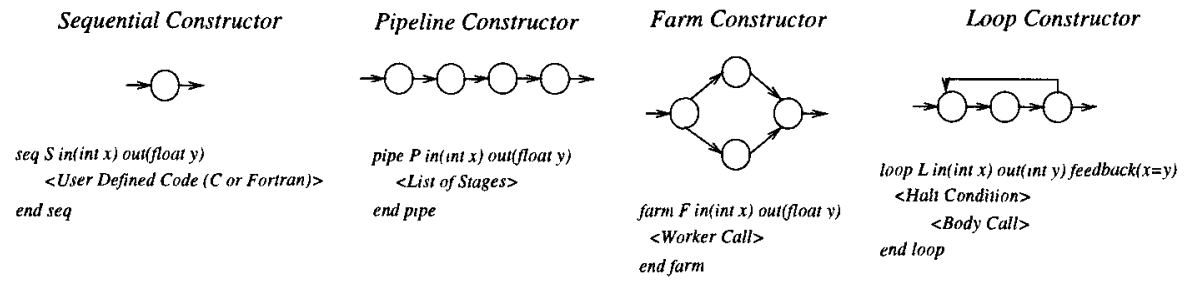

Fig. 1. Stream parallel skeletons representation
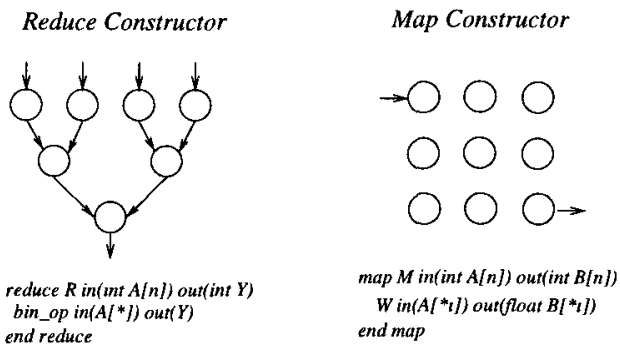

Comp Constructor

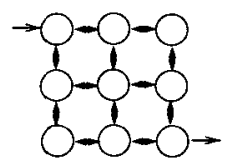

comp C in (int Aln]/m]) out(int B[n]/m]) $<$ List of data parallel constructors $>$ end comp

Fig. 2. Data parallel skeletons representation

computation of the ray tracer on each image $I$ is parallelized using a map constructor decomposing $I$ by row and applying the function worker to each row. Furthermore the map trace is nested in the farm disp that is in charge of dispatching the images to the first map ready to start a new computation on the next stream image.

The composite parallel computation can be viewed as a construct tree, where the leafs correspond to modules assumed as "black box" (information hiding, invariant behavior) and the remaining nodes to instances of the constructs of the coordination language (recursive construction). The root-construct corresponds to the outermost view of the parallel program. Figure 4 shows the tree associated to the program computing the parallel ray tracer on a stream of images.

Host Languages and Interfacing. In principle, the leaf-modules can be written in any language, called host languages; such modules are, possibly complex, programs independently developed by means of their native tools (in-thelarge development of multidisciplinary heterogeneous applications, integration of tools).

The coordination language defines the interfacing rules and mechanisms for the composition, and the data objects which can be passed at the module interfaces [4]. In our approach, these mechanisms and objects must respect the data generality and secure arguments principles for modular construction. The basic semantics of the composition is functional in nature, though any language can be used as host language. The computation is determinate, except where spe- 


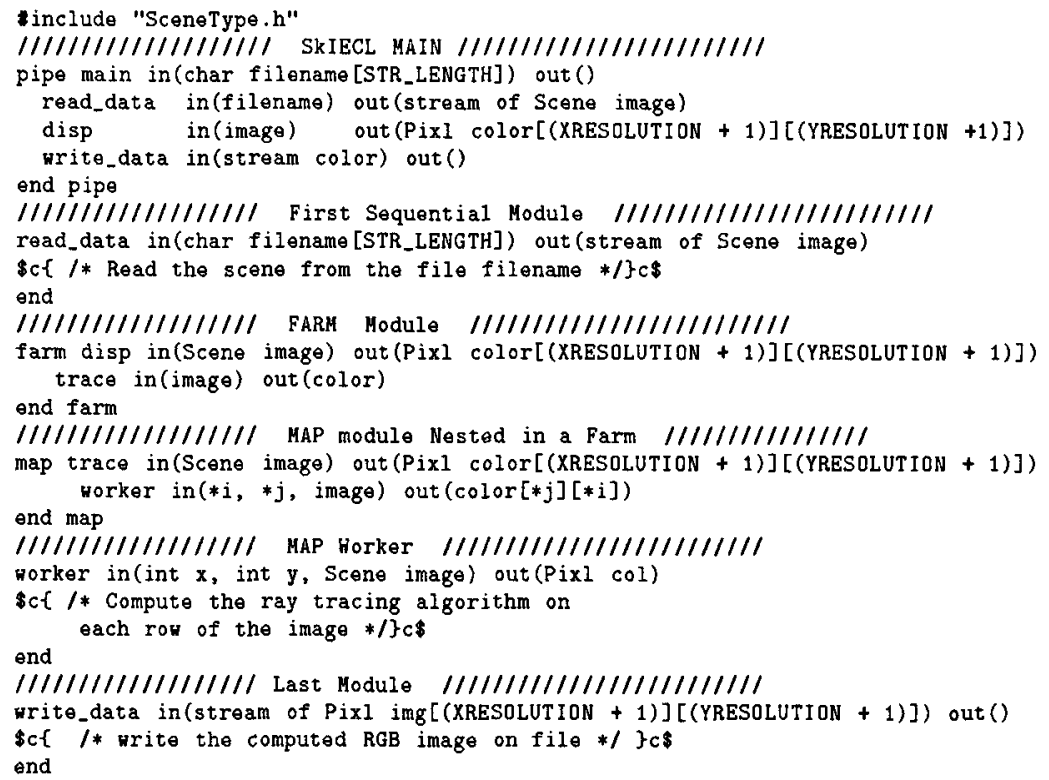

Fig. 3. A SkIECL program computing a ray tracer on a stream of images

cific constructs are used to control constrained forms of nondeterminism (e.g. feedbacks in data-flow loops). As in functional parallel programming, the stream data type is a very powerful mechanisms to define clear and powerful interfaces. Streams are SkIECL data types that allow programmers to manage the input as an unbounded sequence of items. A declaration stream of int a defines a sequence of items each one having type integer and name a. Streams can be nested. Stream can be specialized, that is programmers can specify the constant part that has to be replicated in each element of the stream. Only sequential constructors can create or delete a stream by means of the stream library functions provided by SkIECL language.

Forms of Parallelism: efficiency in irregular computations. The choice of the forms of parallelism corresponding to the skeletons is critical for achieving the best trade-off between modularity, predictability and performance.

The combined usage of data and task parallelism constructs in SkIE is considered fundamental to overcome the limitations of existing SPMD or data parallel standards, notably HPF, in designing irregular computations, i.e. computations in which the synchronization and data patterns, as well as data dependencies, vary dynamically, and dynamic load balancing is often explicitly required to achieve a satisfactory performance level [18]. In SkIECL a very frequent program structure for irregular/dynamic problems consists of parallel loops including stream 


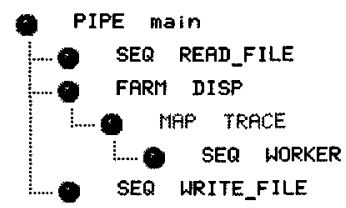

Fig. 4. The Constructor tree to the SkIECL parallel ray tracing

parallel substructures, e.g. farms that guarantee automatic load balancing ("on demand"strategy), and/or pipes of farms; shortly, this kind of computation will be denoted by loop-farm. Loop-farms emulate divide-and-conquer strategies efficiently [23]. In some cases, this structure can be improved by introducing comp and map/reduce in place of farm or at a deeper nesting level inside pipe or farm skeletons. Besides stream parallelism and data partitioning, another feature frequently used in the implementation of irregular/dynamic computations is replication of objects in farming ("specializer" constructor) and in map structures.

A programming model with a performance model. A cost calculus, or performance model, is associated to the coordination language SkIECL. This feature is fundamental in order to implement tools, and in particular the compiler, able to introduce as many global optimizations as possible. In the current implementation, the cost model consists of a set of functions predicting the performance of the template network, according to a set of parameters characterizing the target architecture and the user-defined sequential module code.

An important issue is the predictability of the underlying machine mechanisms, e.g. bandwidth/latency of interconnection structures, performance of memory subsystems, performance of $\mathrm{I} / \mathrm{O}$ and file system. For example the current architecture of PQE2000 adopts an interconnection structure (fat tree) which is highly predictable. When predictability of machine mechanisms is low, the utilization of more powerful performance evaluation tools [22] is of great benefit, provided that they can be really integrated with the tools of the programming environment.

Global Optimizations. In SkIE all the low level issues and the very hard (NP-hard) problems of parallel processing [12] are left to the static and dynamic tools of the coordination language, that applies approximate heuristics to the composition of skeletons. The reader is referred to the literature on $P^{3} L$ for the detailed description of the kind of optimizations adopted by the SkIE compiler. The main features can be summarized as follows:

- the same skeleton can have different implementation templates, among which the compiler can choose the best one for the specific program and target machine; 
- while values of the architecture-dependent parameters are known for a "predictable" abstract/physical machine, the application-dependent parameters are estimated by profiling sequential modules at compile time;

- the construct tree can be optimized according to formal transformations (rewriting rules) applied to the skeleton composition. This is a very promising field in which substantial research has still to be done [19];

- the template/resource optimization phase applies the set of approximate heuristics by descending the construct tree and consulting the performance models and the local optimization rules for each template. This phase assumes an unbounded amount of computing resources;

- to meet the actual amount of computing resources, the implementation is reduced by applying the so called Limited Resources Algorithm [7] that "shrinks" the template network iteratively; at each step the amount of eliminated resources is the one that produces the minimum degradation of performance with respect to the unbounded resources version.

Figure 5 shows the results obtained running the parallel ray tracer, presented in section 3.2 , on a CS2 with 24 nodes. Using the performance models, the SkIECL compiler is able to fix the optimal number of map and farm workers in order to reach the best trade off between efficiency and speed up, with a very good approximation (error less than 10\%)with respect to the measured performances.

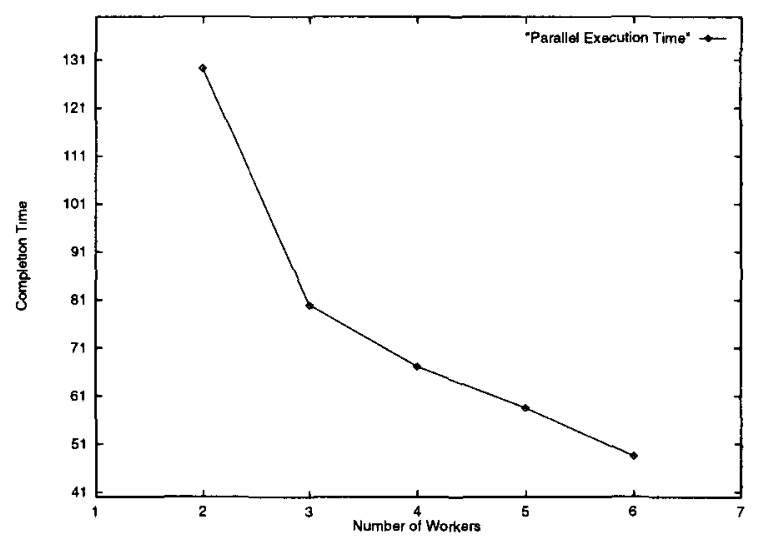

Fig. 5. Execution time of parallel ray tracer (speed up 21, 38 processes generated)

\section{Matching Architecture and Software Technology}

The approach in which the programming model is rigidly derived from the architectural model (or even from a specific architecture) has been, unfortunately, adopted for many years, leading to non-portable, non-modular, very expensive 
and error-prone parallel programs for SIMD and MIMD specific machines. In our opinion, the correct approach is different: the programming model must be able to efficiently exploit the new directions in computer architecture; at the same time, the new directions in computer architecture should be influenced by the general characteristics of the programming models.

Distributed Virtual Shared Memory. Especially in irregular/dynamic computations, skeletons implementations based on a form of shared memory is potentially able to increase the application performance significantly. In the current version of PQE2000, shared memory is emulated, on top of the native hardware, by a component of the Intermediate Virtual Machine called Distributed Virtual Shared Memory (DVSM) $[24,3]$. Its goal is to allow the compiler to choose and to implement the most efficient strategies for shared objects in complex cooperation tasks. As an example, the DVSM implementation of MPI-1 collective operations achieves an improvement by a factor from 3 to 12 with respect to MPI-CH: this is very significant especially if we consider that DVSM is entirely implemented in software. In [25] the performance of irregular/dynamic computations has been evaluated, by using the loop-farm structure with a shared memory implementation using a uniform distribution strategy and excess parallelism in each node to hide memory access latency. It has been shown that significant improvements, of at least one order of magnitude, can be achieved with respect to message passing implementations. The opportunities for optimizations depends on the "by need" access patterns to objects (also a certain amount of fine grain accesses is efficiently tolerated) and by the proper management of the excess parallelism feature. The optimal number of processes per node can be approximated.

Based in these first evaluations, and exploiting the shared memory facilities of PQE2000, work is in progress to deeply evaluate the impact of shared memory implementation of SkIECL ; static and dynamic optimizations will be evaluated with respect to various architectural features (caching, prefetching, interconnection network, multi-threading, dynamic job scheduling, and so on) and language features (e.g., visibility of shared objects at the programmer level).

Memory-Oriented Models. An issue of growing importance is the set of problems caused by the increasing gap between processors and main memory [14] and, consequently, by the fact that access to memory is becoming the main parameter affecting performance. The problem can be dealt with, either on the side of the programming model, or on the side of the architectural model, or both. The SkIE programming environment is, by its own nature, defined in terms of a "performance-driven" programming model. According to the results achieved till now, and to the experiments on new run-time-solutions, we are confident that, given a specific architecture, intensive optimizations can be realized to give a reasonable solution to the memory gap problem. This is due to the features of the coordination language explained in section 3.2 .

If we study the architecture-software technology matching problem from the viewpoint of the parallel architecture, a general trend seems to be common to 
the most interesting architectural models for the next generation: the exploitation of larger amounts of fine grain parallelism. The skeleton model, and SkIECL in particular, has this potential, and this feature doesn't increase significantly the complexity in the application development. Future architectures will be able to exploit the high parallelism of the skeleton model even better. Two main solutions, not mutually exclusive, can be individuated: multi-threading [17] and Processors-In-Memory (PIM) [26,3,2,28]. Their effectiveness to support the efficient implementation of structured parallel programs has to be evaluated accurately.

Another interesting solution to match architecture and software technology is dynamic scheduling in a multiuser context [15]. Dynamic scheduling techniques are fully compatible with the skeleton programming model, and SkIECL in particular. In fact, as sketched in sect. 3.2, the compilation of a SkIE program provides to the allocation and optimization of the available resources through the application of the Limited Resources Algorithm: the same algorithm can be applied at run-time in order to modify the amount of resources allocated to a program, provided that the scheduling strategy maintains the "processor working set" for each job in execution.

Seamless integration of parallel machines into distributed environments. The need for development environments that are much more oriented towards industrial requirements imposes that the characterization of MPP includes distributed systems at local/geographical level, clusters and metacomputers. Many complex industrial processes can be solved by exploiting the full power of all the computational resources available within a corporation, thus adding significant tools for the cooperation in a multidisciplinary environment. Some interesting powerful environments for managing distributed computing resources are emerging, notably at $\mathrm{PAC}$ [1] and Imperial College [16].The programming environment we advocate for parallel machines, being based on the concept of in-the-large application development, is compatible with the requirement of seamless integration of distributed heterogeneous resources. The current version of SkIE contains only basic, state-of-the art features for such integration. Work is in progress to integrate, in the same environment, object-based software infrastructures for integrating distributed MPP servers in complex multidisciplinary applications.

\section{Conclusion}

We have discussed the basic ideas for the development of heterogeneous, multidisciplinary, multi-platform environments for industrial HPC applications. These ideas have been illustrated with reference to the SkIE programming environment of the PQE2000 project, where the research results on parallel programming tools and MPP architectures have been transferred into industrial products at the European level. This experience has demonstrated the feasibility of an approach aiming to achieve a satisfactory trade-off between high-level modular software 
development, high performance, performance portability, rapid prototyping and software reuse, and productivity in a multidisciplinary environment. Moreover, SkIE can act as a solid framework in which the complex set of relationships between new directions in MPP architecture and programming environments can be studied and experimented in the next few years, along with the fundamental issues of integration between object-oriented distributed systems and MPP resources.

\section{Acknowledgments}

The PQE2000 project is the result of the joint efforts of many people I wish to thank collectively. In particular, the following people have done an enormous and qualified work to realize the SkIE programming environment: Marco Danelutto and Susanna Pelagatti, Department of Computer Science, University of Pisa, have coordinated the scientific project of SkIE ; Bruno Bacci, QSW Pisa, has coordinated the design and implementation team composed by: Barbara Cantalupo, Paolo Pesciullesi, Roberto Ravazzolo and Alessandro Riaudo, QSW Pisa, and Marco Aldinucci, Massimo Coppola and Massimo Torquati, Department of Computer Science. Silvia Ciarpaglini and Laura Folchi, INFN fellows at the Department of Computer Science, have contributed to the prototype evaluation of SkIECL compiler. Fabrizio Baiardi, Department of Computer Science, has coordinated the activity on the Intermediate Virtual Machine; in this context, Davide Guerri has realized the first version of DVSM. Domenico Laforenza, CNUCE-CNR Pisa, has coordinated the activity on HPF, with the collaboration of Salvatore Orlando (University of Venice) and Raffaele Perego. I have no words to express my gratitude to all of them. The activity on the software tools of PQE2000 have been supported by the PQE-1 program of QSW and ENEA-HPCN leaded by Agostino Mathis. Thanks are due to Lidia Arcipiani, Paolo Novelli, Vittorio Rosato, Paolo Palazzari, Roberto Iacono of the ENEA Labs, and to Roberto Marega, QSW Rome. Finally, a special thank to Antonio Sgamellotti, University of Perugia, president of the Scientific Committee of PQE2000, to Armando Orlandi, manager director of QSW, and to Roberto Vaccaro, IRSIP-CNR in Naples, for his continuous work to pursue the idea of PQE2000.

\section{References}

1. A.Hey, C.J. Scott, M. Surridge, C. Upstill, "Integrating computation and information resources - an MPP perspective". 3rd International Working Conference on Massively Parallel Programming Models, London, Nov. 12-14, 1997. To be published by IEEE Press.

2. M. Vanneschi, "Variable grain architectures for MPP computation and structured parallel programming. 3rd International Working Conference on Massively Parallel Programming Models, London, Nov. 12-14, 1997. To be published by IEEE Press. 
3. M. Vanneschi, The PQE2000 Project on General Purpose Massively Parallel Systems, PQE2000 Report 96/1, 1996. Also published in Alta Frequenza, IEEE, and in Ercim News.

4. B. Bacci, B. Cantalupo, P. Pesciullesi, R. Ravazzolo, A. Riaudo, "SkIECL User Manual". PQE200 Report, QSW, Pisa, Dec. 997.

5. B. Bacci, M. Danelutto, S. Orlando, S. Pelagatti, M. Vanneschi, "P3L : a structured parallel programming language and its structured support", Concurrency : Practice and Experience, 7 (3), 225-255, May 1995.

6. B. Bacci, B. Cantalupo, M. Danelutto, S. Orlando, D. Pasetto, S. Pelagatti, M. Vanneschi, "An environment for structured parallel programming", Advances in High Performance Computing, Kluwier, The Netherlands, 219-234, 1997.

7. B. Bacci, M. Danelutto, S. Pelagatti, "Resources optimization via structured parallel programming", Programming Environments for Massively Parallel Distributed Systems, Birkhauser-Verlag, 13-25, 1994.

8. S. Pelagatti, "Compiling and supporting skeletons on MPP, 3rd International Working Conference on Massively Parallel Programming Models, London, Nov. 12-14, 1997. To be published by IEEE Press.

9. M. Cole, Algorithmic skeletons : structured management of parallel computation, MIT Press, Cambridge, Mass., 1989.

10. J. Darlington, Y. Guo, H.W. To, Y. Jing, "Skeletons for Structured Parallel Composition", in Proc. of the 15th ACM SIGPLAN Symposium on Principles and Practice of Parallel Programming, 1995.

11. D.B. Skillicorn, Foundations of Parallel Programming, Cambridge University Press, 1994.

12. S. Pelagatti, Structured development of parallel programs "Taylor\&Francis", "London",1998

13. S. Orlando, R. Perego, COLT-HPF, a coordination layer for HPF tasks, University of Venice, Department of Mathematics and Computer Science, TR CS-98-4, March 1998.

14. W.D. Gropp, "Performance driven programming models", 3rd International Working Conference on Massively Parallel Programming Models, London, Nov. 12-14, 1997. To be published by IEEE Press

15. E. Rosti, E. Smirini, L.W. Dowdy, G. Serazzi, K.C. Sevcik, "Processor saving scheduling policies for multiprocrssor systems", IEEE Transaction on Computers 47 (2) 178-189 Febrary 1998 .

16. J. Chattratichat, J. Darlington, Y. Guo, S. Hedvall, M. Koehler, A. Saleem, J. Sutiwaraphun, D. Yang, "A software architecture for deploying high performance solution tio Internet", HPCN Conference, Amsterdam, 1998.

17. J.B. Dennis, "A parallel program execution model supporting modular software construction", 3rd International Working Conference on Massively Parallel Programming Models, London, Nov. 12-14, 1997. To be published by IEEE Press.

18. P. Mehrotra, J. Van Rosendale, H. Zima, "Solving irregular problems with High Performance Fortran", 3rd International Working Conference on Massively Parallel Programming Models, London, Nov. 12-14, 1997. To be published by IEEE Press.

19. S. Gorlatch, C. Lengauer, "Homomorphism (de)composition for parallel programs", 3rd International Working Conference on Massively Parallel Programming Models, London, Nov. 12-14, 1997. To be published by IEEE Press.

20. D. Skillicorn, "Categorical data types", 2nd Workshop on Abstract Machine Models for Highly Parallel Computers, Leeds, UK, April 1993. 
21. W. Kuhn, H. Burkhart, "The Alpstone Project : an overview of a performance modeling environment", 2nd Int. Conf. On HiPC'96, 1996.

22. A. Dunlop, A. Hey, "PERFORM - A fast simulator for estimating program execution time", 3rd International Working Conference on Massively Parallel Programming Models, London, Nov. 12-14, 1997. To be published by IEEE Press.

23. R. Di Meglio, Dynamic computations in massively parallel programming models, PhD dissertation, Department of Computer Science, University of Pisa, March 1994.

24. F. Baiardi, D. Guerri, Op-Memory Library, PQE2000 report, October 1997.

25. M. Torquati, Shared memory support to structured parallel programming paradigms, Computer Science dissertation, Department of Computer Science, University of Pisa, February 1997.

26. T. Sterling, P. Messina, P.H. Smith, Enabling Technologies for Peta(FL)OPS Computing, Caltech Concurrent Supercomputing Facilities, California Institute of Technology, CCSF-45, July 1994.

27. P.M. Kogge, T. Sunaga, H. Miyataka, K. Kitamura, E. Retter, "Combined DRAM and Logic for Massively Parallel Systems", Conference on Advanced Research in VLSI, Chapel Hill, NC, March 1995, pp. 4-16.

28. P. Tenti, Variable grain MIMD/PIM architectures for MPP systems, Computer Science dissertation, Department of Computer Science, University of Pisa, April 1998. 